\title{
Mandenkan
}

MANDENIKAN Bulletin semestriel d'études linguistiques mandé

49 | 2013

Le maninka du Niokolo

\section{Constructions prédicatives spéciales}

Section 8

Denis Creissels

\section{(2) OpenEdition}

Journals

Édition électronique

URL : https://journals.openedition.org/mandenkan/604

DOI : $10.4000 /$ mandenkan.604

ISSN : 2104-371X

Éditeur

Llacan UMR 8135 CNRS/Inalco

Édition imprimée

Date de publication : 1 juin 2013

Pagination : $62-70$

ISSN : 0752-5443

Référence électronique

Denis Creissels, «Constructions prédicatives spéciales », Mandenkan [En ligne], 49 | 2013, mis en ligne le 25 avril 2014, consulté le 16 janvier 2023. URL : http://journals.openedition.org/mandenkan/604 ; DOI : https://doi.org/10.4000/mandenkan.604

Ce document a été généré automatiquement le 16 janvier 2023.

\section{(c) $)(1)(2)$}

Creative Commons - Attribution - Pas d'Utilisation Commerciale - Partage dans les Mêmes Conditions 4.0 International - CC BY-NC-SA 4.0

https://creativecommons.org/licenses/by-nc-sa/4.0/ 


\section{Constructions prédicatives spéciales}

Section 8

Denis Creissels

\subsection{Remarques préliminaires}

1 Le maninka du Niokolo n'a pas de schème prédicatif productif ne faisant intervenir comme termes essentiels que des noms, pronoms ou adjectifs. Mais à côté de la prédication verbale canonique présentée en 4 , qui se caractérise par l'association nécessaire d'un lexème verbal et d'un marqueur prédicatif, le maninka a quelques mots grammaticaux désignés ici comme copules. Ces copules sont incompatibles avec les marqueurs prédicatifs présentés en 4, mais leur rôle dans la construction de la phrase est le même que celui rempli dans la prédication verbale canonique par la combinaison d'un lexème verbal et d'un marqueur prédicatif. Ceci se manifeste d'ailleurs clairement dans le fait que, dès lors qu'on désire exprimer autre chose que la simple assertion d'un état rapporté à une situation qui inclut le moment présent (c'est-à-dire, dans les conditions où des langues comme le français ou l'anglais utiliseraient un verbe 'être' à un temps autre que le présent de l'indicatif), on substitue purement et simplement à la copule une forme fléchie de ke 'devenir' (s'il s'agit de la copule d'identification) ou ta'íra 'se trouver' (s'il s'agit de la copule locative) sans que cela nécessite un quelconque remaniement de la construction.

\subsection{La copule locative be / te}

\subsubsection{Propriétés tonales de la copule locative}

2 La copule locative positive a invariablement le comportement tonal d'un enclitique structurellement dépourvu de ton haut. Ce type de comportement constitue aussi la règle générale pour la copule locative négative, qui toutefois présente dans certaines conditions un comportement tonal exceptionnel. En effet, on observe parfois l'adjonction d'un ton haut sur la syllabe finale du mot qui précède te, ce qui conduit à 
poser l'existence d'une variante 'te. Par exemple, en (56a-b), la réalisation tonale est celle attendue pour un enclitique atone qui en tant que dernière syllabe d'un mot prosodique peut recevoir le ton haut final démarcatif, alors que (56c) implique une forme sous-jacente 'te.

\begin{tabular}{|c|c|c|c|c|c|c|c|}
\hline (56) & a. & Godi & te & $\tilde{n} i^{i}-\bar{y}$ & mo:- & nu & bulu. \\
\hline & & argent & COPN & DEM & personne-DEF & PL & SPHP \\
\hline & & \multicolumn{6}{|c|}{ 'Ces gens n'ont pas d'argent.' } \\
\hline
\end{tabular}

\begin{tabular}{|l|l|l|l|l|l|l|l|l|}
\hline & b. & Godi & té- & $y$ & naí & luntaín-o & lú & bulu. \\
\hline & & argent & COPN & 1 SG & GEN & visiteur-D & PL & SPHP \\
\hline & \multicolumn{6}{|l}{} \\
\hline
\end{tabular}

\begin{tabular}{|l|l|l|l|l|l|}
\hline & c. & Godi: & te & $y$ & bulu. \\
\hline & & argent & COPN & $1 \mathrm{SG}$ & SPHP \\
\hline & & \multicolumn{3}{|l|}{ 'Je n'ai pas d'argent.' } \\
\hline
\end{tabular}

3 Comme on peut le voir d'après cet exemple, aucun facteur syntaxique ou sémantique ne peut expliquer le choix de la variante 'te, qui semble tenir exclusivement à la longueur du complément de la copule : la variante 'te est d'autant plus usuelle que la distance est faible entre la copule négative et la fin de la phrase.

\subsubsection{Be / te et l'expression de la localisation}

Combinée à une expression spatiale (groupe postpositionnel, adverbe, ou groupe nominal apte à fonctionner par lui-même comme adjoint exprimant une localisation), la copule be / te exprime la localisation du référent du sujet.

\begin{tabular}{|l|l|l|l|l|}
\hline$(57)$ & a. & Jòy & be & jée ? \\
\hline & & qui & coploc & là \\
\hline & & \multicolumn{2}{|c|}{ 'Qui est là ?' } \\
\hline
\end{tabular}

\begin{tabular}{|l|l|l|l|l|l|}
\hline & b. & Kúrukesée & be & ñoo & to. \\
\hline & & caillou.D & COPLOC & mil.D & LOC \\
\hline & & \multicolumn{4}{|l|}{ 'Il y a un caillou dans le mil.' } \\
\hline
\end{tabular}




\begin{tabular}{|c|c|c|c|c|c|c|c|c|c|}
\hline c. & 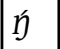 & be & temb-óo & mèy & kono & n-té & be & Kéeduðu & lée. \\
\hline & $1 \mathrm{PL}$ & COPLOC & moment-D & REL & dans & 1SG-EMPH & COPLOC & Kédougou & FOC \\
\hline & 'Er & mor & is & & ou.' & & & & \\
\hline
\end{tabular}

\begin{tabular}{|l|l|l|l|l|l|}
\hline & d. & Móyo & te & lǔw-o & ma. \\
\hline & & personne & CoPN & maison-D & OBL \\
\hline & & \multicolumn{2}{|c|}{ 'Il n'y a personne à la maison.' } \\
\hline
\end{tabular}

\begin{tabular}{|l|l|l|l|l|l|l|l|}
\hline & e. & $\eta$ & $n a a^{-}$ & kuu & te & ñin & to. \\
\hline & 1 SG & GEN & affaire & COPN & DEM & LOC \\
\hline & \multicolumn{6}{|c|}{ 'Je n'y suis pour rien.' litt. 'Aucune affaire à moi n'est pas dans cela.' } \\
\hline
\end{tabular}

5 La copule be / te ne se combine à aucun marqueur prédicatif, mais peut être précédée du marqueur de passé tún.

\begin{tabular}{|l|l|l|l|l|l|l|l|l|l|}
\hline (58) & $y$ & náa-ta & túma & mè, & a & tún & te & lǔw-o & ma. \\
\hline & ISG & venir-ACPP & moment & REL & $3 \mathrm{SG}$ & PAS & COPN & maison-D & OBL \\
\hline \multicolumn{7}{|l}{} \\
\multicolumn{6}{|l}{ 'Quand je suis revenu, il n'était pas à la maison.' } \\
\hline
\end{tabular}

\subsubsection{Omission du complément de be / te}

6 Le complément de la copule locative est en règle générale obligatoire. La seule exception relevée concerne le cas où le sujet désigne un phénomène météorologique, comme à l'ex. (59)'1 .

\begin{tabular}{|l|l|l|l|}
\hline$(59)$ & a. & Nenée & be. \\
\hline & & froid.D & COPLOC \\
\hline & & 'Il fait froid.' & \\
\hline
\end{tabular}

\begin{tabular}{|l|l|l|l|}
\hline & b. & Wu'-ímbée & be. \\
\hline & & chaleur.D & coploc \\
\hline & & 'Il fait chaud.' & \\
\hline
\end{tabular}




\subsubsection{La variante móno de la copule locative négative}

7 Il existe une forme mo même sens, mais qui semble possible exclusivement lorsque le complément est jaín 'ici' ou jecíce 'là'.

\begin{tabular}{|c|c|c|c|c|c|c|c|c|}
\hline (60) & a. & $A$ & te & jacin. & $=$ & $A$ & mo-ino & ja: \\
\hline & & $3 S G$ & COPN & ici & & $3 S G$ & COPN & ici \\
\hline & & \multicolumn{3}{|c|}{ 'Il n'est pas ici.' } & & & & \\
\hline
\end{tabular}

\begin{tabular}{|c|c|c|c|c|c|c|c|}
\hline b. & $A-a_{0}$ & te & Ké-ieduru. & vs. & $*_{A}$ & mo:no & 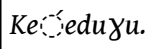 \\
\hline & $3 S G$ & COPN & Kédougou & & $3 S G$ & COPN & Kédougou \\
\hline & \multicolumn{3}{|c|}{ 'Il n'est pas à Kédougou.' } & & & & \\
\hline
\end{tabular}

\subsubsection{Emplois de be / te autres que la localisation spatiale}

Les indications fournies dans cette section ont un caractère purement illustratif, et cette énumération de quelques emplois de la copule locative n'a aucune prétention à l'exhaustivité.

9 Comme c'est généralement le cas en mandingue, l'aspect progressif peut s'exprimer en prenant comme complément de la copule be / te un verbe utilisé nominalement ou un composé $\mathrm{N}+\mathrm{V}$ (où $\mathrm{N}$ exprime l'argument-objet d'un verbe transitif) à la forme déterminée et combiné à la postposition la.

\begin{tabular}{|l|l|l|l|l|l|}
\hline$(61)$ & a. & Jál-ǒo & be & balandin-kos-óo & la. \\
\hline & & griot-D & COPLOC & balafon-frapper-D & OBL \\
\hline & & 'Le griot est en train de jouer du balafon.' \\
\hline
\end{tabular}

\begin{tabular}{|l|l|l|l|l|l|}
\hline & b. & Mús-ǒo & be & kini-tab-óo & la. \\
\hline & & femme-D & COPLoc & riz-cuire-D & овL \\
\hline & & \multicolumn{4}{|c|}{ 'La femme est en train de cuire le riz.' } \\
\hline
\end{tabular}

\begin{tabular}{|l|l|l|l|l|l|}
\hline & c. & Á & be & dólo-mín-o & la. \\
\hline & & 3SG & COPLOC & bière-boire-D & OBL \\
\hline
\end{tabular}


'Il est en train de boire de la bière.'

\begin{tabular}{|l|l|l|l|l|l|l|}
\hline & d. & Á & be & géese-dáa & lée & la. \\
\hline & & $3 \mathrm{SG}$ & COPLOC & fil-tisser.D & FOC & OВL \\
\hline & \multicolumn{4}{|c|}{ 'Il est en train de tisser.' } \\
\hline
\end{tabular}

$\mathrm{Be} /$ te s'emploie aussi pour exprimer le rattachement du référent du sujet à la sphère personnelle d'un possesseur construit comme complément de la copule et marqué de la postposition bulu.

\begin{tabular}{|l|l|l|l|l|l|}
\hline$(62)$ & a. & Godi & te & $y$ & bulu. \\
\hline & & argent & COPN & 1SG & SPHP \\
\hline & & \multicolumn{3}{|l|}{ 'Je n'ai pas d'argent.' } \\
\hline
\end{tabular}

\begin{tabular}{|l|l|l|l|l|l|}
\hline & b. & Dimbayáa & be & $\eta$ & bulu. \\
\hline & & famille.D & COPLOC & 1SG & SPHP \\
\hline & \multicolumn{3}{|l|}{ 'J'ai une famille.' } \\
\hline
\end{tabular}

11 Le caractère épisodique d'une relation de possession peut être souligné en utilisant la postposition kúy à la place de bulu.

\begin{tabular}{|l|l|l|l|l|}
\hline (63) & Godi & te & $\eta$ & kúy. \\
\hline & argent & COPN & $1 \mathrm{SG}$ & SPHP \\
\hline & \multicolumn{4}{|c|}{ 'Je n'ai pas d'argent sur moi.' } \\
\hline
\end{tabular}

La construction avec bulu peut aussi exprimer l'âge.

\begin{tabular}{|l|l|l|l|l|l|l|l|}
\hline (64) & Sañjii & jálu & lée & be & dindig-o & ñí & bulu ? \\
\hline & année & combien & FOC & COPLOC & enfant-D & DEM & SPHP \\
\hline & 'Quel âge a cet enfant ?' \\
\hline
\end{tabular}

13 Avec un complément marqué de la posposition la, la copule locative est d'emploi courant pour exprimer un état affectant un individu. 


\begin{tabular}{|c|c|c|c|c|c|}
\hline (65) & a. & Min-doðóo & be & $\eta$ & na. \\
\hline & & boire-envie.D & COPLOC & $1 S G$ & OBL \\
\hline & & \multicolumn{4}{|l|}{ 'J'ai soif.' } \\
\hline
\end{tabular}

\begin{tabular}{|l|l|l|l|l|l|}
\hline & b. & Konkóo & be & $y$ & na. \\
\hline & & faim.D & COPLOc & $1 \mathrm{SG}$ & ОВL \\
\hline & & \multicolumn{2}{|l|}{ 'J'ai faim.' } \\
\hline
\end{tabular}

\begin{tabular}{|l|l|l|l|l|l|}
\hline & c. & Síinoyóo & be & $y$ & $n a$. \\
\hline & & sommeil.D & COPLOC & 1SG & OBL \\
\hline & & \multicolumn{2}{|l}{} \\
\hline
\end{tabular}

\begin{tabular}{|l|l|l|l|l|l|}
\hline & d. & Mindoyó & te & $y$ & $n a$. \\
\hline & & boire-envie & COPN & 1SG & OBL \\
\hline & & \multicolumn{2}{|l}{} \\
\end{tabular}

\begin{tabular}{|l|l|l|l|l|l|l|}
\hline & e. & Tara-lafiy-o & lée & be & á & la. \\
\hline & & partir-vouloir-D & FOC & COPLOC & 3SG & OBL \\
\hline & & \multicolumn{3}{|l}{} \\
\hline
\end{tabular}

\begin{tabular}{|l|l|l|l|l|l|}
\hline & f. & Godi-kúw-o & bé & $\eta$ & $n a$. \\
\hline & & argent-affaire-D & COPLOC & 1SG & OBL \\
\hline & & \multicolumn{2}{|l}{} \\
\hline
\end{tabular}

\subsection{La copule d'identification $m u$ / tén te}

\subsubsection{La copule d'identification combinée à un seul terme nominal}

Dans cette construction, l'unique terme nominal représente l'identification, laissant implicite ce sur quoi porte l'identification. Lorsque cette construction est utilisée comme phrase assertive indépendante, le focalisateur lée doit obligatoirement être présent. 


\begin{tabular}{|l|l|l|l|l|}
\hline (66) & a. & Yir-óo & lée & mu. \\
\hline & & arbre-D & FOC & COPID \\
\hline & & \multicolumn{2}{|c|}{ 'C'est un arbre.' } \\
\hline
\end{tabular}

\begin{tabular}{|l|l|l|l|l|}
\hline & b. & Daasiyantée & lée & mu. \\
\hline & & bavard-D & FOC & COPID \\
\hline & & \multicolumn{2}{|l|}{ 'C'est un bavard.' } \\
\hline
\end{tabular}

Selon le sens désiré, le focalisateur n'est pas nécessairement inséré entre le terme nominal de la construction et $m u$, mais peut aussi succéder à un modifieur génitival inclus dans ce terme nominal, comme en (67).

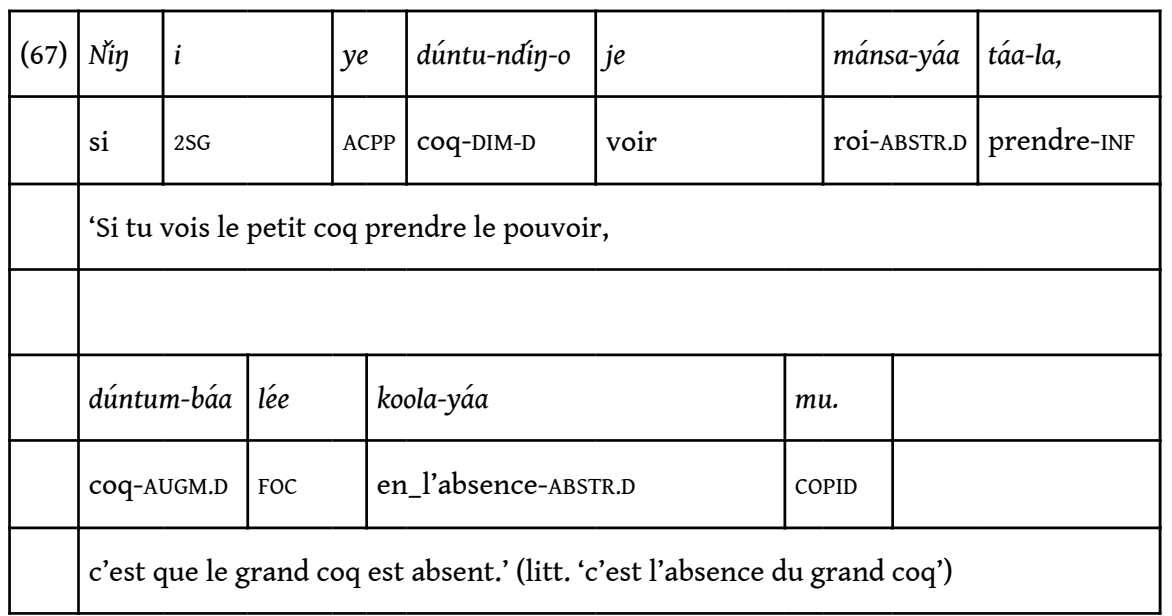

Dans la construction à un seul terme nominal, la copule négative a la forme tén.

\begin{tabular}{|l|l|l|l|}
\hline$(68)$ & a. & Yiri & tén. \\
\hline & & arbre & CopN \\
\hline & & \multicolumn{2}{|c|}{ 'Ce n'est pas un arbre.' } \\
\hline
\end{tabular}

\begin{tabular}{|l|l|l|l|}
\hline & b. & Mo'́ro-soobee & tén. \\
\hline & & personne-sérieux & copN \\
\hline & & \multicolumn{2}{|l}{ 'Ce n'est pas quelqu'un de sérieux.' } \\
\hline
\end{tabular}




\subsubsection{La copule d'identification combinée à deux termes nominaux}

Dans la construction à deux termes nominaux, la copule est suivie d'un groupe nominal marqué de la postposition ti. L'emploi de ti dans cette construction est à relier à la valeur fonctive ('en qualité de') qu'exprime de manière productive cette postposition.

Le terme exprimant l'identification et le terme précisant ce sur quoi porte l'identification peuvent également apparaître comme sujet (précédant la copule) ou comme complément (succédant à la copule et marqué de la postposition ti), mais dans une phrase assertive positive, le terme exprimant l'identication doit être marqué du focalisateur lée.

\begin{tabular}{|l|l|l|l|l|l|l|l|}
\hline (69) & a. & $\eta$ & baadiy-o & lée & $m u$ & $\tilde{n}$ í & ti. \\
\hline & & ISG & parent-D & FOC & COPID & DEM & OBL \\
\hline & & \multicolumn{6}{|l}{} \\
\hline
\end{tabular}

\begin{tabular}{|l|l|l|l|l|l|l|l|}
\hline & b. & A & baabá & $m u$ & jál-ǒo & lée & ti. \\
\hline & & 3SG & père & COPID & griot-D & FOC & OBL \\
\hline & \multicolumn{6}{|l}{} \\
\hline
\end{tabular}

Dans la construction à deux termes nominaux, la copule négative a la forme te.

\begin{tabular}{|l|l|l|l|l|l|}
\hline (70) & a. & Máninká & te & $\tilde{n} i \eta$ & ti. \\
\hline & & Mandingue & COPN & DEM & OBL \\
\hline & & \multicolumn{4}{|c|}{ 'Celui-ci n'est pas Mandingue.' } \\
\hline
\end{tabular}

\begin{tabular}{|l|l|l|l|l|l|}
\hline & b. & Kuu-kende-' & te & ñin & ti. \\
\hline & & problème-bon & COPN & DEM & OBL \\
\hline & & \multicolumn{4}{|c|}{ 'Ce n'est pas vraiment un problème.' } \\
\hline
\end{tabular}

\subsubsection{La copule d'identification et le marqueur de passé}

La copule d'identification ne se combine à aucun marqueur prédicatif, mais peut être précédée du marqueur de passé túy.

\begin{tabular}{|l|l|l|l|l|l|}
\hline (71) & a. & I & lée & túy & mu. \\
\hline
\end{tabular}




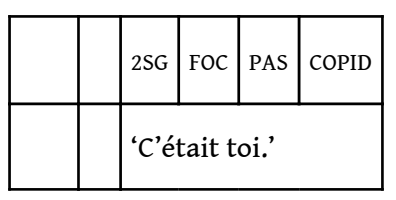

\begin{tabular}{|c|c|c|c|c|c|c|}
\hline b. & Ke & tu & $m u$ & buy-loo-la & leve & ti. \\
\hline & Kékouta & PAS & COPID & case-construire-NMAG.D & FOC & OBL \\
\hline & utc & açor & & & & \\
\hline
\end{tabular}

\subsubsection{Emplois particuliers de la copule d'identification}

Il est possible de construire des phrases exprimant une relation conçue comme une relation d'association en combinant la copule d'identification avec un unique terme nominal incluant une extension associative (cf. section 18).

\begin{tabular}{|l|l|l|l|l|l|l|}
\hline (72) & a. & Á & nì & taða-kúw-o & lée & mu. \\
\hline & & 3SG & avec & partir-affaire-D & FOC & COPID \\
\hline & \multicolumn{6}{|c|}{ 'Il a envie de partir.' lit. 'C'est lui avec l'affaire de partir.' } \\
\hline
\end{tabular}

\begin{tabular}{|l|l|l|l|l|l|l|}
\hline & b. & A & nì & taráa & lée & mu. \\
\hline & & 3SG & avec & partir.D & FOC & COPID \\
\hline & \multicolumn{6}{|c|}{ 'Il a envie de partir.' lit. 'C'est lui avec le départ.' } \\
\hline
\end{tabular}

Il est possible aussi de construire la copule d'identification avec un infinitif en ' $k a \sim$ ' $k e$ pour signifier le caractère souhaitable de l'événement encodé par l'infinitif.

\begin{tabular}{|l|l|l|l|l|l|l|}
\hline (73) & a. & Ka & ñin & tímba & lée & mu. \\
\hline & & INF & DEM & frapper & FOC & COPID \\
\hline & \multicolumn{5}{|c|}{ 'Celui-ci mérite d'être frappé.', 'Il n'y a qu’à le frapper.' } \\
\hline
\end{tabular}

\subsection{Les présentatifs fele et je}

Les présentatifs fele et je, qui historiquement résultent d'une réanalyse de l'impératif des verbes fele 'regarder' et je 'voir', se rencontrent dans des constructions semblables à la prédication d'identification décrite en 8.3. Ils expriment en principe une valeur ostensive ('voici / voilà'), mais s'observent aussi dans des contextes où on peut les 
considérer comme de simples variantes du prédicatif d'identification $m u$. Les séquences fele ñí ti et fele wóo ti se réalisent usuellement [fèlénti] et [fòló:ti].

\begin{tabular}{|l|l|l|l|l|l|l|l|}
\hline (74) & a. & $\eta$ & dì-o & lui & fele & ñin & ti. [fèlénti] \\
\hline & & $1 \mathrm{SG}$ & enfant-D & PL & voici & DEM & OBL \\
\hline & \multicolumn{6}{|l}{ 'Voici mes enfants.' } \\
\hline
\end{tabular}

\begin{tabular}{|l|l|l|l|l|l|l|l|}
\hline & b. & $\eta$ & dì-o & lú: & fele & wóo & ti. [fòló:ti] \\
\hline & & $1 S G$ & enfant-D & PL & voici & DEM & OBL \\
\hline & & \multicolumn{7}{|c|}{ 'Voilà mes enfants.' } \\
\hline
\end{tabular}

\begin{tabular}{|l|l|l|l|l|l|l|}
\hline (75) & a. & A & la & músóo & lée & je. \\
\hline & & $3 S G$ & GEN & femme.D & FOC & voici \\
\hline & \multicolumn{5}{|c|}{ 'Voici sa femme.' } \\
\hline
\end{tabular}

\begin{tabular}{|l|l|l|l|l|l|l|l|}
\hline & b. & I & la & dábǎa & je & wóo & ti. \\
\hline & & 2 SG & GEN & houe.D & voici & DEM & OBL \\
\hline & \multicolumn{6}{|c|}{ 'Voici ta houe.' } \\
\hline
\end{tabular}

\subsection{Diina 'vouloir'}

Le sens de 'vouloir' peut s'exprimer au moyen du verbe régulier lai-ífii, qui se construit intransitivement avec comme complément, ou bien un constituant nominal marqué de la postposition la, ou bien un groupe verbal à l'infinitif, ou bien une phrase au subjonctif.

\begin{tabular}{|l|l|l|l|l|l|}
\hline (76) & a. & A & láfii-tá & god-óo & la. \\
\hline & & $3 \mathrm{SG}$ & vouloir-ACPP & argent-D & OBL \\
\hline & & \multicolumn{3}{|c|}{ 'Il veut de l'argent.' } \\
\hline
\end{tabular}

\begin{tabular}{|l|l|l|l|l|l|l|}
\hline & b. & A & láfii-ta & wúl-ǒo & lu & funtí-ndin-na. \\
\hline & & $3 \mathrm{SG}$ & vouloir-ACPP & chien-D & PL & sortir-CAUS-INF \\
\hline
\end{tabular}


'Il veut faire sortir les chiens.'

\begin{tabular}{|l|l|l|l|l|l|l|}
\hline & c. & A & láfii-ta & $\grave{y}$ & ge & funti. \\
\hline & & $3 S$ SG & vouloir-ACPP & $1 \mathrm{PL}$ & SUBJP & sortir \\
\hline & \multicolumn{5}{|c|}{ 'Il veut que nous sortions.' } \\
\hline
\end{tabular}

Mais la même signification peut s'exprimer au moyen de diina, qui peut être décrit comme un verbe irrégulier. Comme les copules, diina assume la fonction prédicative sans avoir à se combiner à un marqueur prédicatif. Il se construit avec comme complément une phrase au subjonctif.

\begin{tabular}{|l|l|l|l|l|l|l|l|l|l|}
\hline$(77)$ & $y$ & diina & lée & $i$ & yé & tayá & $\eta$ & yáa & biii. \\
\hline & $1 \mathrm{SG}$ & vouloir & FOC & 2SG & SUBJP & aller & $1 \mathrm{SG}$ & chez & aujourd'hui \\
\hline
\end{tabular}

Ce verbe irrégulier est probablement à rapprocher étymologiquement du terme emprunté à l'arabe diina 'religion', attesté ailleurs en mandingue avec le sens de 'caractère, humeur', et il est raisonnable de penser qu'il partage cette étymologie avec la préposition diina 'plus que', 'plutôt que' (cf. 16.5, 22.13).

\section{NOTES}

1. Cette possibilité d'emploi de la copule locative sans complément est un des traits qui distinguent le maninka du Niokolo du mandinka. On doit noter toutefois que la même signification s'exprime de façon apparemment plus courante, soit avec le verbe ke 'avoir lieu' à la

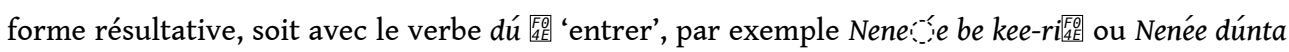
'Il fait froid'. 


\section{AUTEUR}

DENIS CREISSELS

Université de Lyon

Denis.Creissels@univ-lyon2.fr 